\title{
Operative or Interventional Treatment in Infrainguinal Bypass Occlusion: Are There Predictive Factors Affecting Outcome?
}

\author{
T. Betz ${ }^{1}$, C. Uhl ${ }^{1}$, M. Steinbauer ${ }^{1}$, N. Zorger ${ }^{2}$, I. Töpel ${ }^{1}$ \\ ${ }^{1}$ Department of Vascular Surgery, Krankenhaus Barmherzige Brüder Regensburg, Regensburg, Germany \\ ${ }^{2}$ Department of Radiology, Krankenhaus Barmherzige Brüder Regensburg, Regensburg, Germany \\ Email: thomas.betz@barmherzige-regensburg.de
}

Received August 16, 2012; revised September 29, 2012; accepted October 10, 2012

\begin{abstract}
Purpose: To evaluate outcome of interventional and surgical treatment in infrainguinal bypass occlusion and to identify predictive factors affecting therapeutic success. Material and Methods: Over a period of three years 96 patients with infrainguinal bypass occlusion were included in this retrospective study. 52 patients were treated by catheter-directed thrombolysis and 44 patients by reconstructive vascular surgery. Results: 41 grafts could be reopened in thrombolytic group (78.8\%), thrombolysis failed in 11 cases (21.2\%). 34 grafts were treated successfully by reconstructive vascular surgery (77.3\%), 10 grafts couldn't be reopened or reoccluded within 30 days after surgical therapy. After a median observation time of 14.7 months 30 of 48 (62.5\%) bypass grafts treated with intraarterial thrombolysis and 19 of 27 (70.4\%) grafts treated with thrombectomy were overall patent. Limb salvage was $81.3 \%$ in thrombolytic group and $88.8 \%$ in surgery group. No predictive factors affecting outcome could be identified in both groups except the localization of the occluded graft (above/below knee). Conclusion: Infrainguinal bypass occlusion is a serious and challenging complication in vascular surgery. Our study showed similar results for both therapeutic strategies. Despite our data was retrospective with a small number of patients the localization of the occluded graft as predictive pre-therapeutic factor was significant in thrombolytic and surgery group. Based on these scientific findings we established a guideline for choice of therapeutic treatment in our institution after searching current literature.
\end{abstract}

Keywords: Thrombolytic Therapy; Bypass Occlusion; Percutaneous Intervention; Bypass Thrombectomy

\section{Introduction}

Long-term patency rates of infrainguinal bypass grafts are-depending on the localization of the graft and the graft material—satisfying. But critical limb ischemia due to acute bypass occlusion remains a challenging and serious problem, which threatens both, the patients limb and life [1].

Two therapeutic strategies - surgical revascularization or catheter directed intraarterial thrombolysis-are well established today with similar amputation and mortality rates [2]. If thrombolysis is successful it is a non-invasive procedure to restore bypass flow. Not only the bypass graft, but also outflow and collateral vessels are reopened. The causative lesion is unmasked and can be treated by angioplasty or stent placement. If surgical intervention is still necessary, it can be performed on a well-prepared patient on an elective basis [3]. Sometimes choice of therapeutic treatment seems to be difficult. Thus several factors predicting outcome must be considered by the clinician.

\section{Material and Methods}

\subsection{Baseline Characteristics}

Using the database of our institution we identified 96 patients with infrainguinal bypass occlusion treated in our department of vascular surgery between June 2008 and August 2011. During this period 52 patients underwent intraarterial catheter directed thrombolysis. Patients with an acute graft occlusion (symptoms less than 14 days) and a threatened or viable limb as defined by the guidelines for acute lower extremity ischemia were included. Baseline characteristics and clinical conditions of these patients are listed in Table $\mathbf{1 .}$

44 patients with severe ischemic limbs with sensory loss and muscle weakness were considered unsuitable for thrombolysis and treated by reconstructive vascular surgery; patients with absolute contraindications for intraarterial thrombolysis, too. Before starting thrombolysis a duplex ultrasound of the ipsilateral and contralateral iliac and femoral vessels was performed to show any vessel stenosis affecting puncture. 
Table 1. Baseline characteristics of Patients with infrainguinal bypass occlusion.

\begin{tabular}{|c|c|c|}
\hline Baseline characteristics & $\mathbf{n}$ & Percentage \\
\hline Age (mean) & 69 & \\
\hline Gender (M/F) & $68 / 28$ & $70.8 \% / 29.2 \%$ \\
\hline \multicolumn{3}{|c|}{ Medical comorbidities } \\
\hline Smoking history & 32 & $33.3 \%$ \\
\hline Coronary artery disease & 66 & $68.7 \%$ \\
\hline Hypertension & 85 & $88.5 \%$ \\
\hline Diabetes mellitus & 42 & $43.7 \%$ \\
\hline Hypercholesterolemia & 77 & $80.2 \%$ \\
\hline \multicolumn{3}{|c|}{ Prethrombosis antiplatelet therapy } \\
\hline Aspirin only & 62 & $64.6 \%$ \\
\hline Clopidogrel only & 1 & $0.01 \%$ \\
\hline Combination of aspirin and clopidogrel & 3 & $0.03 \%$ \\
\hline Warfarin/coumarin & 26 & $27.1 \%$ \\
\hline Combination of coumarin and aspirin & 4 & $0.04 \%$ \\
\hline \multicolumn{3}{|c|}{ Symptoms at admission (Fontaine classification) } \\
\hline Stadium IIb & 14 & $14.6 \%$ \\
\hline Stadium III & 58 & $60.4 \%$ \\
\hline Stadium IV & 24 & $25 \%$ \\
\hline \multicolumn{3}{|c|}{ Bypass characteristics } \\
\hline Above-knee femoropopliteal bypass & 51 & $53.1 \%$ \\
\hline Below-knee femoropopliteal bypass & 15 & $15.6 \%$ \\
\hline Infrapopliteal bypass & 30 & $31.3 \%$ \\
\hline Venous & 19 & $19.8 \%$ \\
\hline PTFE & 69 & $71.9 \%$ \\
\hline Composite & 8 & $8.3 \%$ \\
\hline
\end{tabular}

\subsection{Technique}

After percutaneous puncture of the contralateral femoral artery and introduction of an arterial sheath a diagnostic angiography was performed in crossover technique. The occluded graft was passed by a guide wire and a multiside hole catheter was placed into the clot after guide wire transversal test. If the bypass origin couldn't be found or the occluded graft could'nt be passed the procedure was aborted.

Standard thrombolysis protocol consisted of a bolus of urokinase (250,000 IE) and then a continous overnight infusion $(100,000 / h)$ under close surveillance in the intermediate care unit. Simultaneously unfractionated heparin in low dose was applicated over the arterial sheath (15,000 25,000 IU/24 h). After 24 hours a control angiograph was performed. Successful lysis was defined as restoration of antegrade blood flow and complete clot lysis (>90\% volume). Any causative lesions demasked by thrombolysis were treated with immediate PTA or surgical revision within a few days. In case of surgical treatment graft thrombectomy with intraoperative angiography was performed. Causative lesions (e.g. anastomotic stenosis) were treated by adjunctive therapy (patch. intraoperative angioplasty. new bypass graft). After successful therapy unfractionated heparin was administered for $48 \mathrm{~h}$ intravenously aiming a partial thromboplastin time of 60 - $80 \mathrm{~s}$. Patients achieved a duplex ultrasound for documenting graft patency prior to discharge.

\section{Results}

During this period 96 patients (68 males, 28 females, mean age 69, range 53 - 86 years) with infrainguinal graft 
occlusion underwent therapy. 14 patients presented with claudication (14.6\%). 58 patients with limb-threatening ischemia and rest pain (60.4\%) and 24 patients with a gangrene or necrosis at the foot (25\%). 51 above-knee femoropopliteal bypasses (53.1\%), 15 below-knee femoropopliteal bypasses (15.6\%) and 30 infrapopliteal bypasses $(31.3 \%)$ were treated by thrombolysis or surgery. There were 19 vein grafts (19.8\%), 69 PTFE grafts (71.9\%) and 8 composite grafts (8.3\%).

41 grafts could be reopened by thrombolysis (78.8\%). In 15 cases intraarterial thrombolysis was successful without adjunctive therapy (36.6\%). In 26 cases flow limiting lesions-hämodynamically significant stenosis or residual thrombus were unmasked (63.4\%): 9 lesions were treated by surgery (21.9\%) and 17 by angioplasty (41.5\%). Thrombolysis failed in 11 cases (21.2\%). Of these 3 patients declined any further therapy. 1 patient received a bypass thrombectomy. 5 patients a new bypass graft and 2 patients a major amputation due to persistent limb threatening ischemia. In these 2 cases any surgical revascularization was not possible. Thrombolysis related complications were seen in 8 cases $(15.4 \%)$. 3 patients developed an acute renal failure (5.8\%) immediately after thrombolysis. one patient had a major groin hematoma $(1.9 \%)$ and required blood transfusion and one developed a wound infection at the puncture site and needed antibiotic treatment (1.9\%); one was operated on a false aneurysma after thrombolysis (1.9\%). Mortality was 5.8\%. Two patients died a few days after thrombolysis propably due to myocardial infarction (no post mortem examination was performed).

34 from 44 grafts were treated successfully by surgery (77.3\%). Thrombectomy alone was sufficient in 6 cases (17.6\%). In 28 cases (82.4\%) underlying causative lesions. e.g. anastomotic stenosis were unmasked and treated by patch (proximal or distal anastomosis) (20.5\%) or angioplasty alone (20.5\%) or patch in combination with an angioplasty (8.8\%). In case of persistent occlusion blood flow was restored by jump bypass or new bypass graft (32.3\%). 2 bypass grafts couldn't be reopened. Reocclusion occurred in 6 cases in the first 30 days. 2 patients died a few days after thrombectomy; one due to acute myocardial infarction and one due to persistent limb ischemia with progredient ischemia and palliative therapy. After a median observation time of 14.7 months (1 - 56 months) 4 further patients in thrombolysis group and 5 patients in thrombectomy group were deceased. 30 of 48 (62.5\%) bypass grafts treated with intraarterial thrombolysis overall were patent. Primary patency in thrombolytic group was $46.7 \%$. 16 patients (53.3\%) needed a reintervention. 12 patients received surgical treatment due to reocclusion ( 6 new bypass grafts, 2 jump bypasses, 4 patches) and 9 patients a major amputation. 4 patients needed a percutaneous transluminal angioplasty.
3 patients were spoiled at time of assessment. Overall Limb salvage was $81.3 \%$. After median observation time 19 of 27 (70.4\%) grafts were overall patent in thrombectomy group. Primary patency was 33.3\%. 10 patients needed reintervention (37.4\%). 5 further patients were deceased at time of assessment. 2 were spoiled. Overall limb salvage was $88.8 \%$. Altogether 11 from 96 patients (11.5\%) received a major amputation. 13 patients were deceased (13\%) and 5 patients were lost to follow-up at time of assessment.

To evaluate an association between patient demographics and comorbid conditions on thrombolytic or surgical success we performed a Chi-Test with our data (Tables 2 and 3). Statistical analysis detected a significant association between failed or successful thrombolysis and thrombectomy and graft localization above or below-knee.

\section{Discussion}

Acute infrainguinal bypass occlusion is a serious complication in vascular surgery. Several studies had been performed to end ongoing debate when to choose thrombolysis and when to choose surgery in case of acute limb ischemia. Most studies are retrospective with a small and heterogenous number of patients. Only three large prospective randomized studies had been performed in the mid-nineties which confirmed that thrombolysis is an alternative to surgical therapy with similar amputation and mortality rates [4,5]. However choice of treatment often seems to be difficult in daily routine.

Despite our data was retrospective with a small number of patients our study showed that the localization of the occluded graft as predictive pretherapeutic factor was significant in thrombolytic and surgery group. It seems reasonable that therapeutic success depends on the localization of the occluded graft. The better "outflow-section”, the better the outcome. Below knee grafts had poorer outflow vessels. normally. From our experience it is reasonable to treat "simple" above-knee grafts by surgery. More complex tibial or pedal reconstructions or patients who had already been operated should be treated by intraarterial catheter directed thrombolysis. Other predictive factors like gender, age, preexisting diseases, antiplatelet therapy and graft material were not significant [6]. So we searched literature after additional predictive factors affecting therapeutic outcome.

Thrombolyis only seems to be successful in a defined time frame. This is due to the fact that presence of vessel thrombus causes damage to the vessel endothelium and longer ischemic intervals (longer than 14 days) are associated with more extensive endothelial injury and increasing thrombogenicity [7]. If the clinical symptoms last longer than 14 days a thrombolytic therapy doesn't seem to be reasonable $[2,5,8]$. Because intravascular clot is organized and adherent at the graft wall. Age of the 
Table 2. Association between successful thrombolysis and prethrombolytic factors in 52 patients treated with intraarterial thrombolysis.

\begin{tabular}{ccc}
\hline \multicolumn{1}{c}{ Thrombolytic outcome versus } & Chi-Test: p-values \\
\hline Male & Female & 0.281 \\
$<70$ years & 70 years $>$ & 0.859 \\
Claudication & Rest pain & 0.178 \\
Smoker & Non-smoker & 1.000 \\
Hypercholesterolemia & Normal cholesterol level & 0.668 \\
Diabetics & Non-diabetics & 0.721 \\
Graft localization above knee & Below-knee & 0.044 \\
Venous graft & PTFE graft & 0.329 \\
Renal insufficiency & Normal renal function & 0.331 \\
ASs & Phenprocoumon & 0.419 \\
\hline
\end{tabular}

Table 3. Association between successful thrombectomy and preoperative factors in 44 patients treated with surgery.

\begin{tabular}{ccc}
\hline \multicolumn{1}{c}{ Outcome after Thrombectomy versus } & Chi-Test: p-values \\
\hline Male & Female & 0.557 \\
$<70$ years & 70 years $>$ & 0.126 \\
Claudication & Rest Pain & 0.255 \\
Smoker & Non Smoker & 0.557 \\
Hypercholesterolemia & normal cholesterol level & 0.445 \\
Diabetics & Non-diabetics & 0.601 \\
Graft localization above knee & below knee & 0.031 \\
Venous graft & PTFE graft & 0.678 \\
Renal insufficiency & Normal renal function & 0.968 \\
ASS & Phenprocoumon & 0.459 \\
\hline
\end{tabular}

bypass graft and the graft material might be relevant for thrombolytic success. too. Vein grafts longer than one year in place had better long term patency after thrombolysis [9]. Older grafts are better incorporated into the connective tissue and the nutrient supply depends not only on the intraluminal blood flow but also on the ingrown adventitial capillaries. So vessel endothelium compensates "thrombolytic damage" better. However long-term patency of vein grafts after thrombolysis is poorer than patency of prosthetic grafts. Thrombotic occlusion causes sclerosis with thrombotic organization and endothelial necrosis of the vein graft [10]. Thrombus of a occluded prosthetic graft is less adhesive and inner surface will be slower remodeled and obturated by connective tissue. Based on these scientific findings we developed a guideline for choice of thrombolytic treatment in our institution (Flowchart Table 4) and established a scoring system to assess thrombolytic success: patients presenting with limb ischemia due to infrainguinal bypass occlusion should be first clinically examined to evaluate the clinical category. A duplex ultrasound must be performed to detect any vessel stenosis above or below the occluded bypass graft. Patients with sensory loss muscle paralysis and inaudible Doppler signals (clinical category Rutherford III) shouldn't be treated by thrombolysis and need immediate surgical therapy. Exclusion criteria for thrombolysis must be checked: patients with absolute contraindications for intraarterial thrombolysis, including severe uncontrolled hypertension, stroke or transient ischemic attack within two months, history of internal, gastronintestinal bleeding or major surgery within two weeks must be excluded from thrombolytic therapy [4]. Before starting thrombolytic therapy prethrombolytic situation should be checked (duration of clinical symptoms, age of 
Table 4. Guideline for choice of thrombolytic treatment.

\begin{tabular}{|c|}
\hline Infrainguinal bypass occlusion \\
$\qquad \downarrow$ \\
\hline Clinical examination and Duplex ultrasound \\
\hline
\end{tabular}

\section{$\kappa$ \\ Clinical category $>$ Rutherford IIb}

$\downarrow$

Exclusion criteria

\section{Clinical category Rutherford III}

$\downarrow$

\section{Surgical treatment}

1.Profound ischemia of the involvd limb resulting in neurologic abnormality sufficient to cause permanent motor paresis and sensory loss 2.Severe uncontrolled hypertension with sustained systolic pressure of $180 \mathrm{~mm} \mathrm{Hg}$ or greater or diastolic pressure of $110 \mathrm{~mm}$ Hg or greater

3. History of cerebral vascular accident within the previous 6 months or transient ischemic attacks within the previous 2 months

4.Significant internal bleeding within 10 days

5.History of serious gastrointestinal bleeding.major surgery,biop sy of organs,puncture of non compressible vessels.or serious trauma within 14 days 6.Severe hepatic dysfuncyion

7.Any condition in which surgical intervention,intraarterial thrombolytic therapy,or angiography is contraindicated

8.Life expectancy less than 1 year

Ouriel and Veith. Acute lower limb ischemia: Determinants of outcome. Surgery. Vol 124, Nr.2, $336-342$

$\downarrow$

No exclusion criteria

\section{Thrombolytic treatment}

$\downarrow$

Before starting therapy check prethrombolytic situation

\begin{tabular}{|l|l|l|}
\hline & Yes & No \\
\hline Clinical symptoms lasting shorter than 14 days & & \\
\hline Venous graft older than one year & & \\
\hline Prosthetic graft & & \\
\hline Above knee localization of bypass graft & & \\
\hline $\begin{array}{l}\text { Yes: } 1 \text { point, No: } 0 \text { point, } 4 \text { points thrombolytic success most likely, with diminishing value high risk for } \\
\text { therapy failure }\end{array}$ & \\
\hline
\end{tabular}

Prethrombolytic situation $\geq 2$ points

K

Start thrombolytic therapy

\section{$\rightarrow \quad$ Surgical treatment}


bypass graft, bypass material, graft localization). A scoring system helps to assess therapeutic outcome (Yes: 1 point, No: 0 point, 4 points thrombolytic success most likely, with diminishing value high risk for therapy failure).

\section{REFERENCES}

[1] E. B. Dreglid, et al., "Patient Survival and Limb Prognosis after Arterial Embolectomy," European Journal of Vascular Surgery, Vol. 1, No. 4, 1987, pp. 263-271. doi:10.1016/S0950-821X(87)80078-6

[2] A. J. Comerota, F. A. Weaver, J. D. Hosking, et al., "Results of a Prospective, Randomized Trial of Surgery versus Thrombolysis for Occluded Lower Extremity Bypass Grafts,” American Journal of Surgery, Vol. 172, No. 2, 1996, pp. 105-112. doi:10.1016/S0002-9610(96)00129-8

[3] K. Ouriel, et al., “Acute Peripheral Arterial Occlusion: Predictors of Success in Catheter-Directed Thrombolytic Therapy,” Radiology, Vol. 193, No. 2, 1994, pp. 561-566.

[4] K. Ouriel and F. J. Veith, "Acute Lower Limb Ischemia: Determinants of Outcome,” Surgery, Vol. 124, No. 2, 1998, pp. 336-342

[5] STILE Investigators, "Results of a Prospective Random- ized Trial Evaluating Surgery versus Thrombolysis for Ischemia of the Lower Extremity. The STILE Trial,” Annals of Surgery, Vol. 220, No. 3, 1994, pp. 251-266. doi:10.1097/00000658-199409000-00003

[6] J. C. van den Berg, "Thrombolysis for Acute Arterial Occlusion,” Journal of Vascular Surgery, Vol. 52, No. 2, 2010, pp. 512-515.

[7] M. F. Conrad, et al., "Long-Term Results of CatheterDirected Thrombolyis to Treat Infrainguinal Bypass Graft Occlusion: The Urokinase Era," Journal of Vascular Surgery, Vol. 37, No. 5, 2003, pp. 1009-1016. doi:10.1067/mva.2003.176

[8] R. K. Greenberg and K. Ouriel, "A Multi-Modal Approach to the Management of Bypass Graft Failure," Vascular Medicine, Vol. 3, No. 3, 1998, pp. 215-220. doi:10.1177/1358836X9800300306

[9] H. D. Berkowitz and J. C. Kee, “Occluded Infrainguinal Grafts: When to Choose Lytic Therapy versus a New Bypass Graft," The American Journal of Surgery, Vol. 170, No. 2, 1995, pp. 136-139. doi:10.1016/S0002-9610(99)80272-4

[10] C. K. Shorttell and K. Ouriel, "Thrombolysis in Acute Peripheral Arterial Occlusion: Predictors of Immediate Success,” Annals of Vascular Surgery, Vol. 8, No. 1, 1994, pp. 59-65. doi:10.1007/BF02133407 